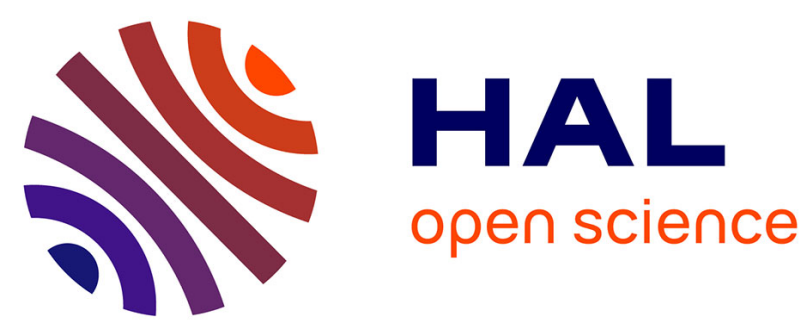

\title{
Comparison of a kaleidoscope-based multi-view infrared system with its TOMBO-based counterpart
}

\author{
Adrien Mas, Guillaume Druart, Maxime Vaché, Sylvain Favier, Aymeric \\ Alazarine, Eric Compain, Nathalie Morin, Nicolas Guérineau
}

\section{To cite this version:}

Adrien Mas, Guillaume Druart, Maxime Vaché, Sylvain Favier, Aymeric Alazarine, et al.. Comparison of a kaleidoscope-based multi-view infrared system with its TOMBO-based counterpart. SPIE Security \& Defence 2019, Sep 2019, Strasbourg, France. 10.1117/12.2532808 . hal-02613804

\section{HAL Id: hal-02613804 \\ https://hal.science/hal-02613804}

Submitted on 20 May 2020

HAL is a multi-disciplinary open access archive for the deposit and dissemination of scientific research documents, whether they are published or not. The documents may come from teaching and research institutions in France or abroad, or from public or private research centers.
L'archive ouverte pluridisciplinaire HAL, est destinée au dépôt et à la diffusion de documents scientifiques de niveau recherche, publiés ou non, émanant des établissements d'enseignement et de recherche français ou étrangers, des laboratoires publics ou privés. 


\title{
Comparison of a kaleidoscope-based multi-view infrared system with its TOMBO-based counterpart
}

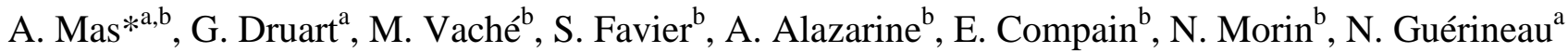 \\ ${ }^{a}$ Département Optique et Techniques Associées, ONERA, Université Paris Saclay F- 91123 \\ Palaiseau, France ; ${ }^{\mathrm{b}}$ Bertin Technologies, 155 Rue Louis Armand ZI, 13290 Aix-en-Provence, France
}

\begin{abstract}
Multi imaging snapshot systems are used for a wide range of applications in all the spectral ranges. We propose here a study and a realization of a multi-view snapshot system using a kaleidoscope in the Long-Wave Infrared (LWIR) and compatible with uncooled infrared detectors such as microbolometers. The optical system has a high numerical aperture, a wide range of fields of view and uses a single focal plane array. We will establish here the advantages of this technology on other design strategies and especially the kaleidoscope design will be compared with the TOMBO design. Then the optical conception rules for every subset of the kaleidoscope architecture will be described and the results of a first demonstrator will be presented. The features of this system will be compared with a TOMBO-based system with a front afocal.
\end{abstract}

Keywords: Multi-view, infrared, microbolometer, kaleidoscope

\section{INTRODUCTION}

The ability to acquire several images simultaneously on a single focal plane array is a useful function for a wide range of applications such as polarization, miniaturization, super-resolution, 3D imagery and spectral imagery. Spectral imagery can have applications in gas detection, and it is in this context that Bertin Technologies and ONERA have launched research work on the design of a multi-spectral snapshot camera.

Currently, Bertin Technologies offers an uncooled multispectral camera in the LWIR for gas detection applications. The current product is called Second Sight and is presented in Fig. 1. This camera allows imaging a scene in six distinct spectral bands using a filter wheel and thus allows the detection, recognition and quantification of a gas cloud. However, spectral images are taken one after the other, which can affect the camera's detection sensitivity, especially if the scene changes quickly or if the camera is mobile. The objective of this paper is to overcome this problem by developing a camera that simultaneously takes several images of the scene under different spectral bands and on a single focal plane array.

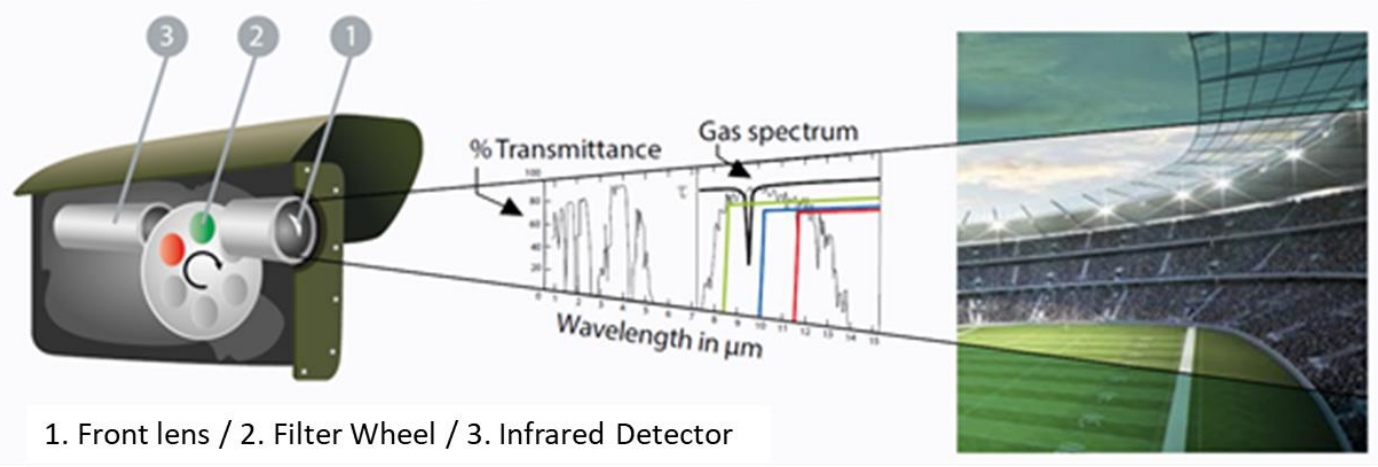

Fig. 1. Principle of detection of the Second Sight 
We are first looking for a multi-view design with good spatial resolution for pixel co-registration and fields of view ranging from 10 to 60 degrees, what the Second Sight can do by changing its front lens. We intend to use uncooled infrared detectors in the Long Wavelength Infrared spectral range (LWIR) based on microbolometers and we will thus focus on infrared designs able to produce multiple images on a single Focal Plane Array (FPA). Indeed, since the signal from a microbolometer can easily drift with temperature, it is therefore suitable to use a single FPA to process different images since each image will drift in the same way. Moreover, when using microbolometers, we need a high numerical aperture to maximize the signal to noise ratio, typically an aperture around F/1.

Different optical architectures have been proposed to meet these new spectral imagery needs. Thus, multi-aperture, single-aperture or hybrid concepts have been studied ${ }^{1}$. Several architectures based on the integration field spectrometry (IFS) concept, so called because each individual measurement of a datacube pixel results from integrating over a region of the field, have already been studied. To divide the field of view, several techniques were used: with mirrors (IFS-M) ${ }^{2}$, lens arrays (IFS-L) $)^{3}$ or optical fiber bundles (IFS-F) ${ }^{4}$. Each field of view is then spectrally dispersed. However, these architectures tend to strongly limit spatial resolution or numerical aperture in favor of spectral resolution whereas we do not think we need more than 10 spectral bands for our application. Moreover, these designs don't give the choice of the spectral bandwidth of each channel. In the Second Sight camera, high-pass and low-pass filters are used.

Another concept is the multi-aperture filtered camera ${ }^{5}$ which uses an array of imaging elements, such as an array of cameras or a monolithic lenslet array, with a different filter placed at each element in order to collect portions of the full spectral band. A LWIR uncooled TOMBO camera, has already been made ${ }^{6}$. TOMBO concept has the advantage of being extremely compact and making a large number of images with few optical components aligned. However, we will see later that, with our specifications, these architectures can have a major disadvantage.

Light-field type cameras were studied too ${ }^{7,8}$. Among these different concepts the single aperture light-field design based on a kaleidoscope ${ }^{9,10}$ seems interesting and has a field stop that prevents any crosstalks between the subimages. The principle of this optical architecture is to place between a first imaging lens and a re-imaging one, a kaleidoscope which is a tube made of 4 plane mirrors. An intermediate image is formed by the first lens at the entrance of the kaleidoscope. By reflection in the kaleidoscope, the cones of incident beams are divided into $\mathrm{N} \times \mathrm{N}$ cones with different orientations that form virtual copies of the image produced by the first lens at the intermediate image plane. This array of subimages is then focused by the re-imaging lens on the FPA. In Manakov's paper ${ }^{9}$, a diffuser is used at the entrance of the kaleidoscope to burst the cone of incident beams and to send it on the mirrors of the kaleidoscope. So, a large number of subimages can be formed independently of the aperture of the front lens and off-the-shelf lenses can be used. However, a diffuser induces serious loss of light and image quality and this approach is thus not adapted for LWIR systems using an uncooled detector. In Manakov's paper ${ }^{9}$, authors also proposed a light-field imaging system without diffuser by adding a field lens next to the entrance of the kaleidoscope. However, in Manakov's paper ${ }^{9}$, the use of off-the-shelf lenses makes this approach difficult and the authors uses a long kaleidoscope resulting in a decrease of the numerical aperture of the whole system.

In this paper, we highlight drawbacks of classic multi-aperture concept when a high aperture is required and propose a kaleidoscope architecture inspired from Manakov and Pacheco's work ${ }^{9,10}$ which exceeds the multi-aperture limitations. In this paper, we demonstrate that a kaleidoscope concept can be adapted to meet the light efficiency constraints of uncooled infrared detectors. We demonstrate the FOV- F-number uncoupling capacity of this system and we finally compare the kaleidoscope architecture with a TOMBO architecture coupled with an afocal that is used to adjust the field of view of the whole camera.

\section{DESIGN RULES OF THE TOMBO MULTI-APERTURE DESIGN}

One of the most classic designs for achieving multiple images on a single detector is the TOMBO multi-channel architecture $^{5}$ which has been widely studied. This concept uses an array of imaging elements such as a monolithic lenslet array, to juxtapose several optical systems and to produce these multiple images on a single FPA, as presented in Fig. 2, where NA is the Numerical Aperture of the optical channel. This results in a relationship between the F-number and the Field Of View (FOV) to avoid either a blind area on the detector or a field overlap between adjacent channels. Indeed, to maximize the number of useful pixels while having the smallest F-number, FOV (in radian) and F-number are linked in first approximation by the following equation, explained in more details in the article [11]:

$$
F \#=\frac{1}{2 \times N A}=\frac{1}{F O V}
$$




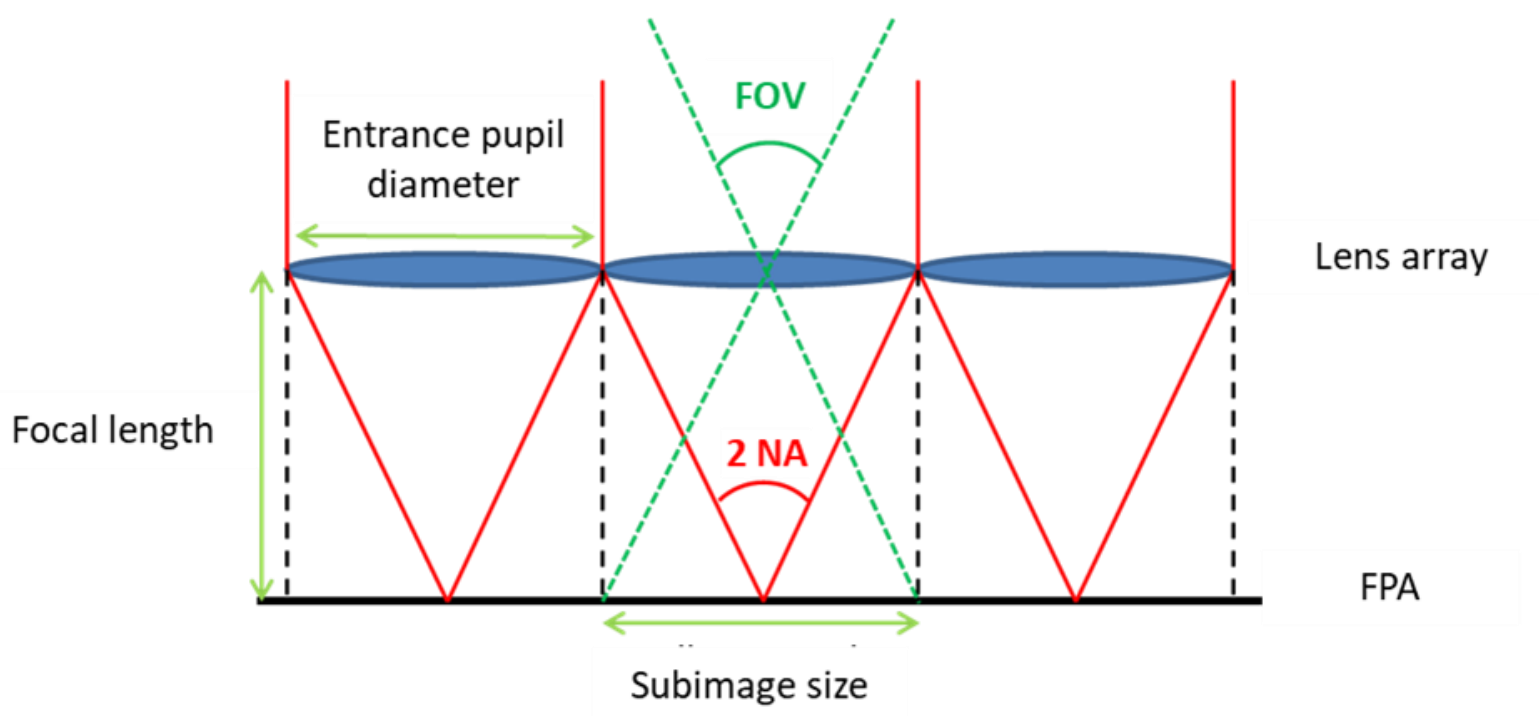

Fig. 2. Concept of a TOMBO architecture

Therefore, a narrow field of view with a high numerical aperture induces areas of unused pixels on the FPA. A LWIR uncooled TOMBO camera has already been made $^{6}$, and the size of an image with the desired field of view is smaller than the channel size. Thus, since the lenses cannot be physically superimposed on each other, the result is an area of unused pixels between the subimages of each channel as shown in Fig. 3. In this figure, a TOMBO camera with two lenses by channel is used and the FOV is $30^{\circ}$ for a F-number of 1.2 where the unused pixels between the channel can be clearly seen. Indeed, according to the previous equation, it would require a F-number of 1.5 to avoid pixel loss.

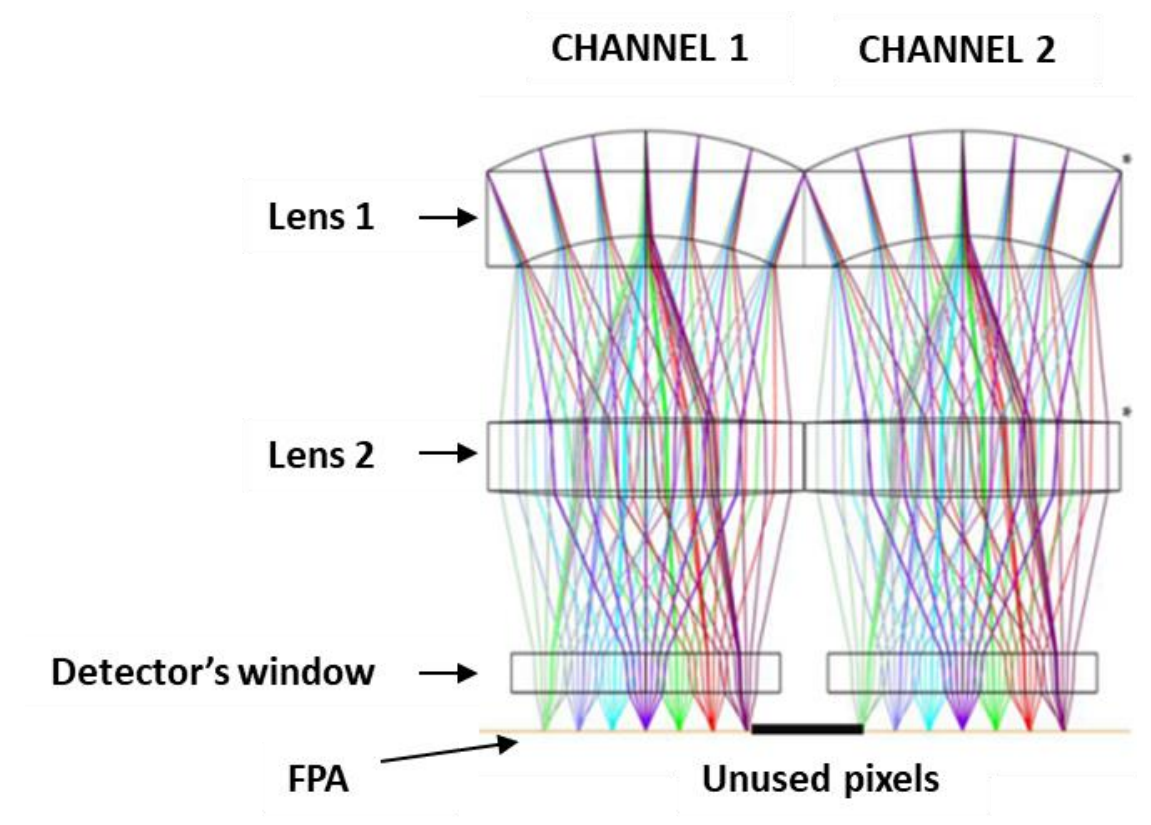

Fig. 3. Simulation with the optical design software Zemax of a two-stage TOMBO for a Field Of View of $30^{\circ}$ and an aperture of F/1.2. Unused pixels area appears between two subimages. Aspheric surfaces are marked by*.

To remove the area of unused pixels while having a high numerical aperture of F/1, it would require a FOV of $52^{\circ}$. Such performance is hardly achievable with a two-lens TOMBO system as illustrated in Fig. 4 A and B. In Fig. 4 B the MTF for some fields of view of a two-lens TOMBO system are given. We notice that even with highly aspheric surfaces, the 
performance of the system is not closed to the limit of diffraction. In contrary, good performance can be achieved by adding a third lens to the optical channel and by using highly aspheric surfaces as presented in Fig. $4 \mathrm{C}$ and D. In Fig. 4 $\mathrm{D}$ the MTF for some fields of view of a three-lens TOMBO system are given. The performance of this system is closed to the limit of diffraction. Moreover, removing all the unused pixel areas involves making square subimages, which requires a higher field of view to complete the diagonal of the subimages. In our previous example, it would require a diagonal FOV around $72^{\circ}$, which is not achievable for a system so constrained on the diameter of its lenses.
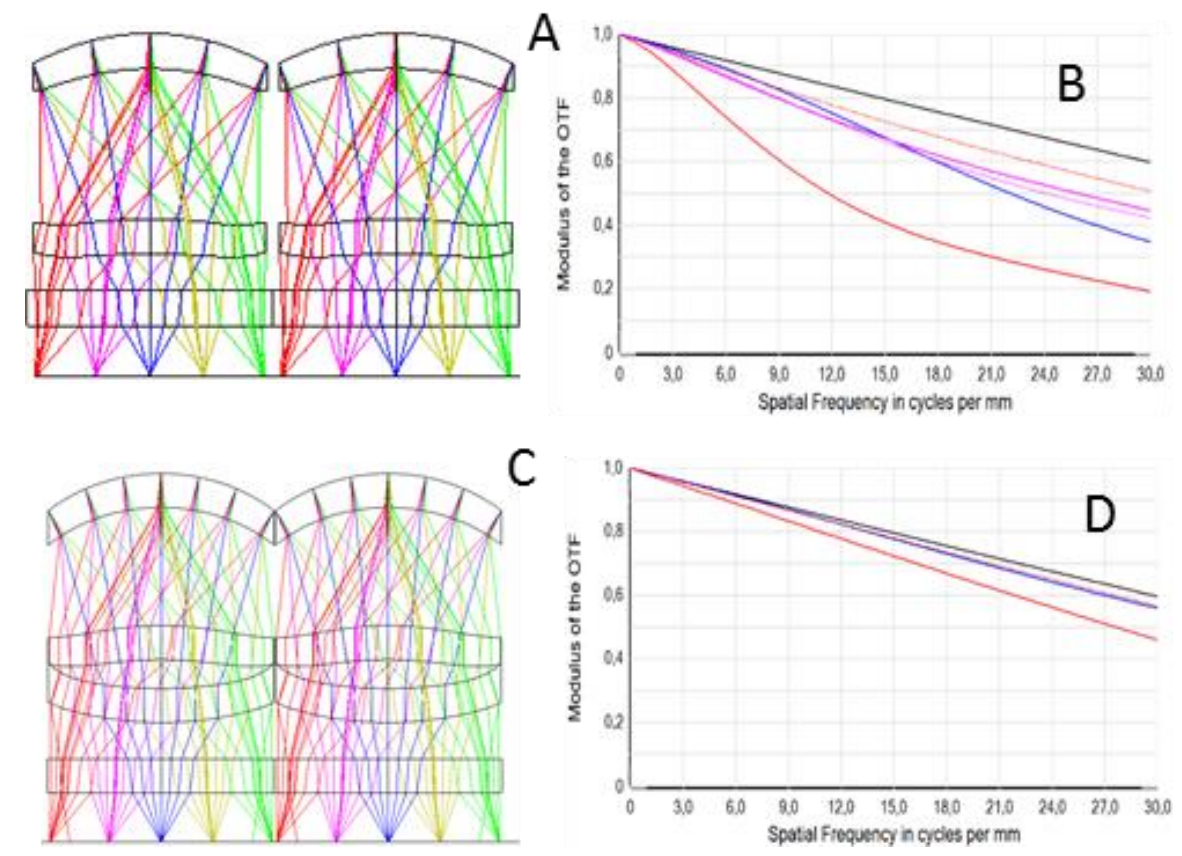

Fig. 4. Two multi-aperture architecture with an aperture of F/1 and for a horizontal FOV of $52^{\circ}$. In A) a two-stage solution is presented with its associated FTMs curves for some fields of view in B). In C) a three-stage solution is presented with its associated FTMs curves for some fields of view in D).

To overcome the constraint of F-Number/FOV coupling of equation (1), Carles's team proposed a two-stage freeform TOMBO architecture allowing an increase of the numerical aperture by using off-axis optics to tilt the optical axis of each channel ${ }^{12}$. Freeform surfaces are used to correct the induced off-axis aberrations generated by this approach. With this design, the unused pixel area can be reduced for small field of view systems (in Carles'paper ${ }^{12}$, the field of view is equal to $8^{\circ}$ but with a F-number of only 1.6). However, freeform surfaces that can be different from one channel to another increase drastically the complexity of the optical system. Since unconventional lenses have to be made and aligned, this solution is difficult to apply. Finally, multichannel architectures must manage crosstalk between the different channels, usually by using walls, since a field stop cannot be easily implemented for high numerical aperture ${ }^{13}$. We conclude from the state of the art that TOMBO-like multi-aperture concepts have limitations for high aperture systems with narrow FOVs.

\section{DESIGN RULES OF THE KALEIDOSCOPE-BASED CAMERA}

The kaleidoscope architecture is composed of 3 parts:

- A front lens that will make the image of the scene at the entrance plane of the kaleidoscope.

- A kaleidoscope, whose mirrors will reflect this image and transform this image into an image array.

- A re-imaging lens that will image the image array on the FPA.

The principle of this camera is illustrated in the Fig. 5. Since the intermediate image is located at the entrance of the kaleidoscope, this entrance section is used as a field stop and its section defines the shape of the images. Moreover, this 
field stop prevents any crosstalks between the different subimages, which is an advantage of this architecture. Our kaleidoscope camera is designed to make $3 \times 3$ subimages of the scene on the FPA. In our array of $3 \times 3$ subimages, the central subimage corresponds to the rays that pass through the kaleidoscope without being reflected (highlighted by green rays in Fig. 5). The 4 side subimages are the subimages constructed with a single reflection (two of these are highlighted by red and purple rays in Fig. 5), and the diagonal subimages are due to a double reflection on two adjacent mirrors of the kaleidoscope (not represented in Fig. 5). If the kaleidoscope-based multi-view design is properly designed, all the light entering the kaleidoscope is equally divided in these 9 subimages. This means that there is no reflection on opposite mirrors.

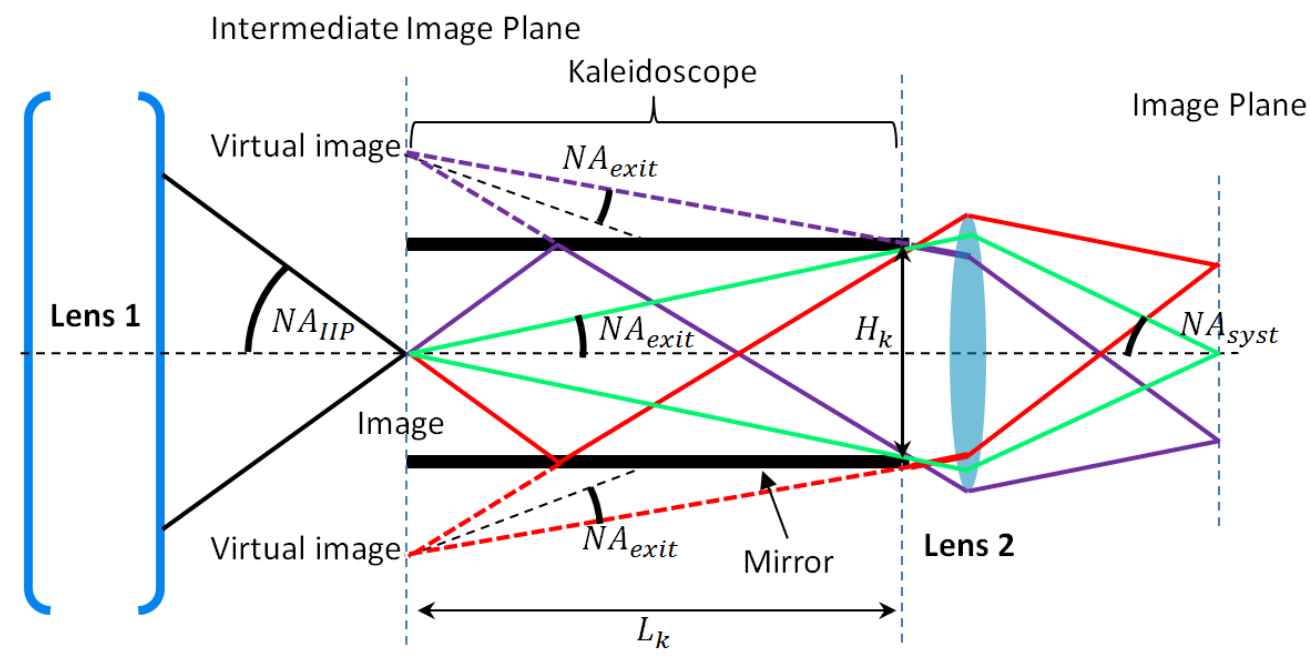

Fig. 5. Scheme of a kaleidoscope camera architecture. $\mathrm{NA}_{\mathrm{IIP}}$ is the numerical aperture of the front lens, $\mathrm{NA}_{\text {exit }}$ the numerical aperture at the kaleidoscope exit and $\mathrm{NA}_{\text {syst }}$ the numerical aperture of the whole system.

Our kaleidoscope-based architecture has been designed under the optical design software Zemax. It is illustrated in Fig.6. In Fig. 6.A, some rays of the central field are represented to show the image points created in the different subimages. In Fig. 6.B, rays of two more extreme fields are added to show the layout of the subimages on the uncooled detector. Both the front lens and the reimaging lens are composed of 3 lenses. Fig. 6.A shows that the cones of rays on the FPA have the same angles as the cone of rays on the intermediate image plane. Thus, if the front lens is opened at F/1, the whole kaleidoscope-based system is opened at F/1 as well. Indeed, despite the fact that the kaleidoscope divides the entrance pupil of the system by $3 \times 3$, thus reducing by 9 the amount of light allocated to each subimage compared to the amount of light allocated to the intermediate image, a well-chosen re-imaging lens reduces the focal length of the system by 3 compared to the focal length of the front lens. By this way, the subimages have a surface area 9 times smaller than the intermediate image and the numerical aperture of the whole system is remained constant compared to he numerical aperture of the front lens. Finally, this architecture is modular since the front lens can be replaced by another one if we want to change the FOV. The kaleidoscope and the reimaging lens can thus be considered as a generic multi-view module for a given F-number and a given size of a focal plane array. 


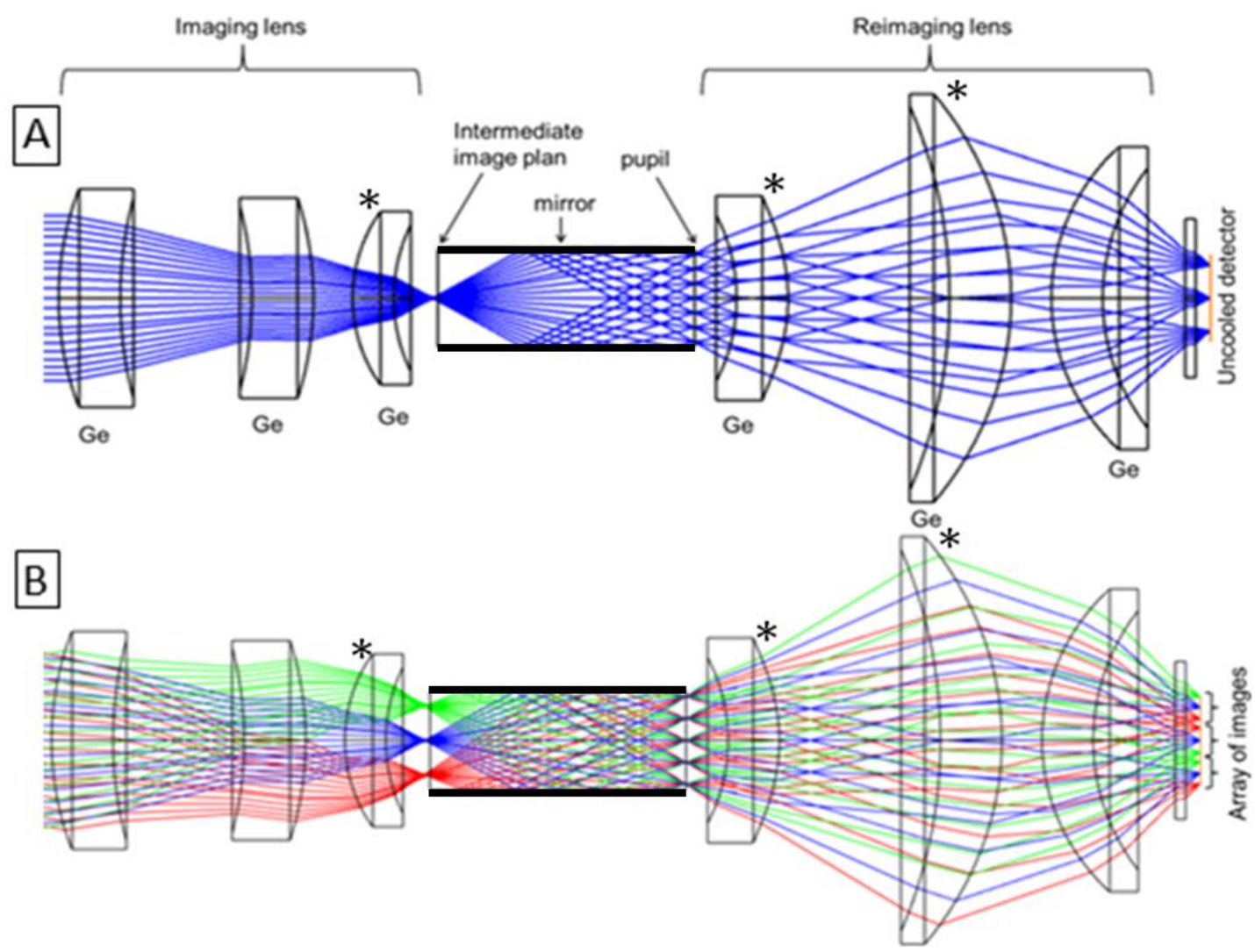

Fig. 6. Final infrared multi-view architecture based on a kaleidoscope and adapted for a microbolometer. Aspheric surfaces are marked with $*$.

\section{CHARACTERIZATION OF THE KALEIDOSCOPE-BASED DEMONSTRATOR}

According to the optical design illustrated in Fig. 6, we have made a first kaleidoscope-based infrared optical system. We wanted to limit as much as possible the number of optics and the number of aspheric surfaces to ease the manufacturing of this system. Thus, the system could be easily improved optically, to the detriment of manufacturing simplicity and optical alignment. Finally, we converged to the demonstrator illustrated in Fig. 7. It is opened at F/1 and has a field of view of $30^{\circ}$. The kaleidoscope has a square section of $9 \mathrm{~mm}$ and is $25 \mathrm{~mm}$ long. The reimaging lens has a magnification close to $1 / 3$ and the multi-imaging system produces $3 \times 3$ images. The FPA chosen is an uncooled $640 \times 480$ detector with a pixel pitch of $25 \mu \mathrm{m}$. The total length of the optical system is $110 \mathrm{~mm}$ for a focal length of $6.5 \mathrm{~mm}$. 


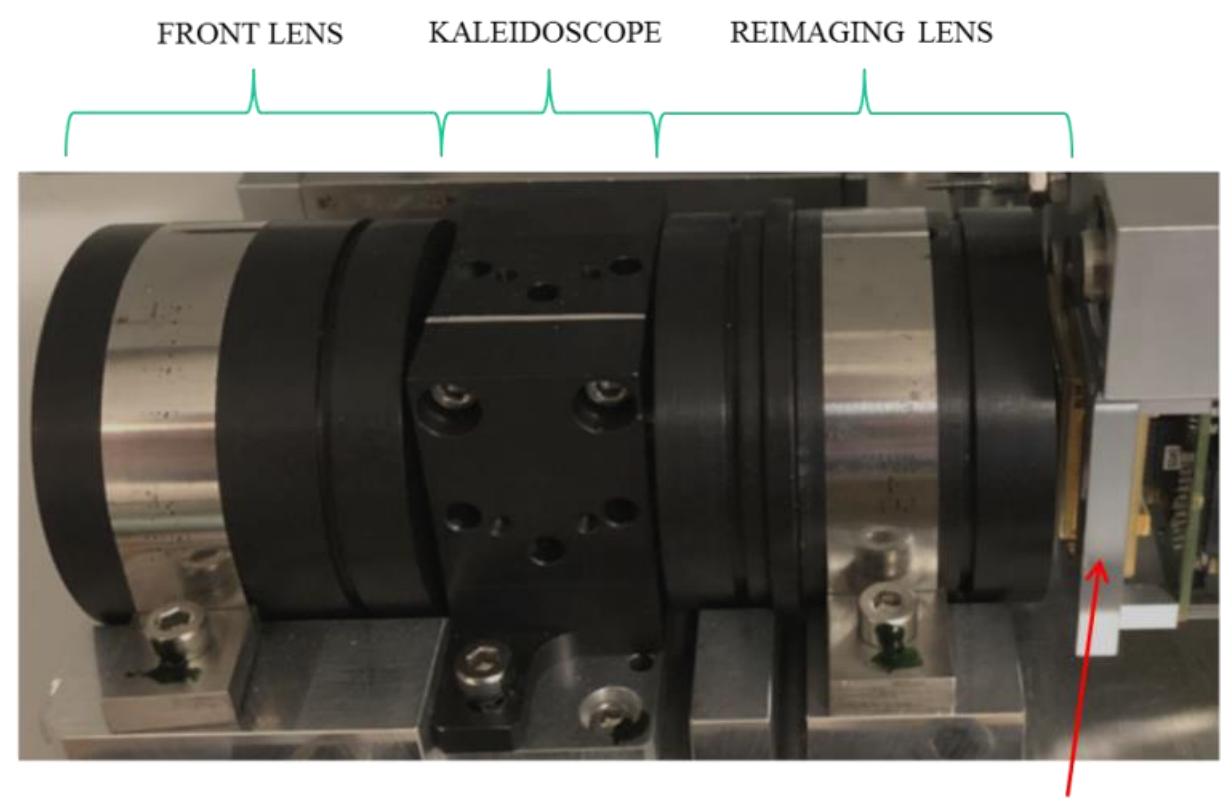

Uncooled LWIR detector

Fig. 7. Demonstrator of the infrared kaleidoscope-based multi-view system.

Images obtained from this demonstrator are illustrated in Fig. 8 . We clearly see $3 \times 3$ subimages: a central subimage and 8 others with orientations that match one or two reflections inside the kaleidoscope. The image on the right shows electrical wires and corrugated iron roof. A zoom on the central subimage of this picture is given in Fig. 9. It highlights a good image quality and a good radiometric performance, which is possible due to the high aperture of the system. There are no areas of unused pixels between the subimages because the image array observed on the detector is created by the reflections of the intermediate image on the mirrors of the kaleidoscope.
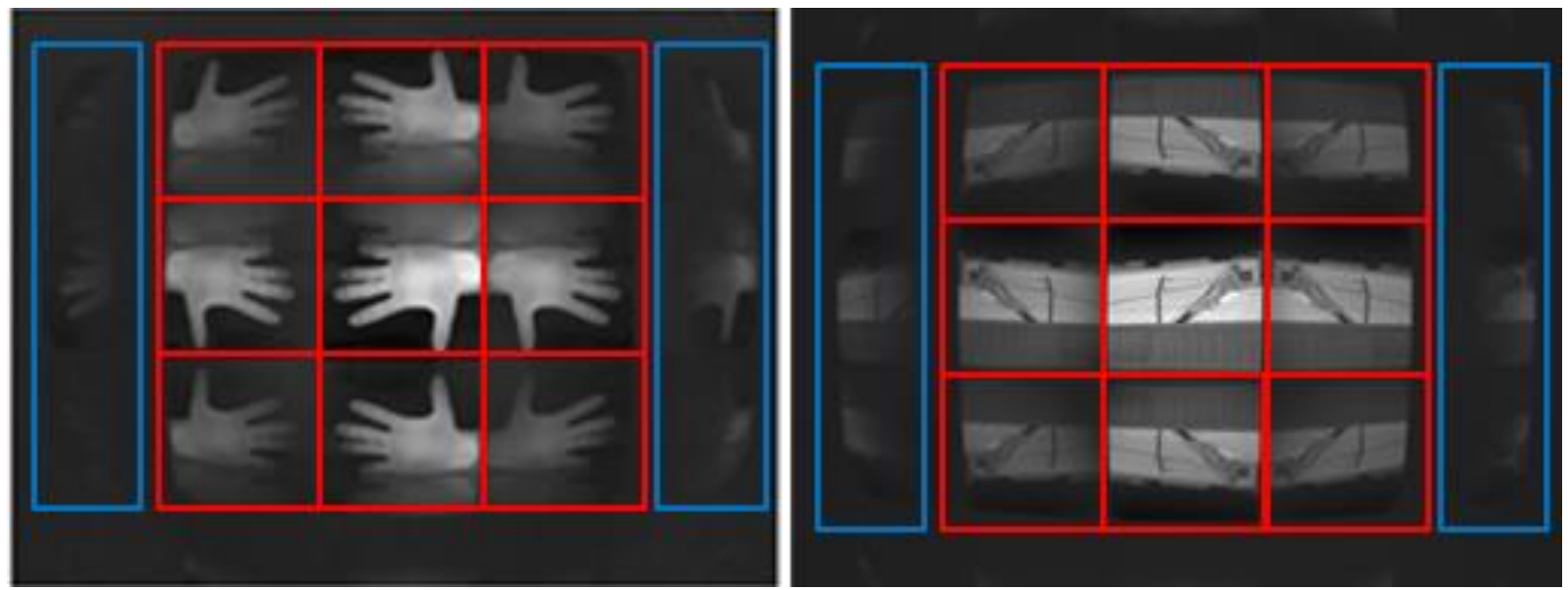

Fig. 8. On the left, an image of a hand seen through the kaleidoscope-based system which clearly shows orientations of each 9 subimages (framed in red); on the right, an image of a roof where we can distinguish small details like an overhead electrical wire that highlights the image quality of the system. Extra images resulting from double reflections on opposite mirrors are framed in blue.

In Fig. 8, we can notice extra images resulting from double reflections on opposite mirrors which are due to an entrance pupil a bit higher than expected. Indeed, in our demonstrator, the aperture of the front lens that should be opened at F/1 
wasn't correctly fixed. Thus, the numerical aperture of the rays entering the kaleidoscope is higher than expected and double reflections on opposite mirrors can occur.

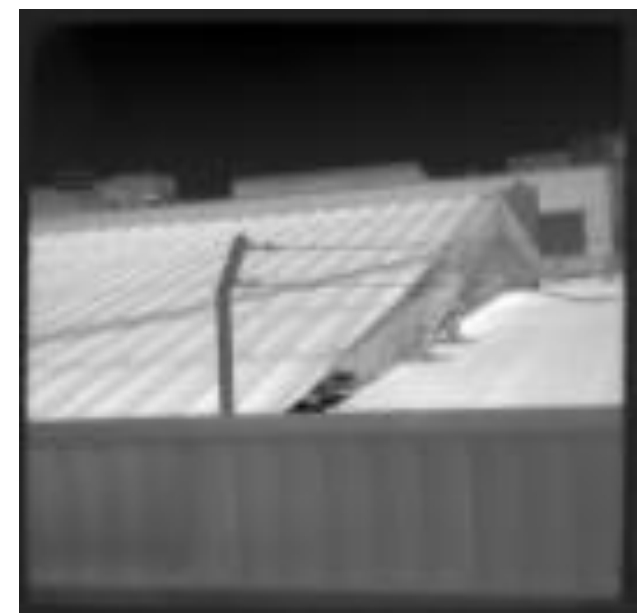

Fig. 9. Zoom on the central subimage of the kaleidoscope camera. We can see details showing a good image quality and a good radiometric performance.

We also measured the demonstrator's Modulation Transfer Functions (MTFs) to compare them with the theoretical MTFs given by Zemax software. These curves are shown in Fig. 10. They show a clear difference between the theoretical and experimental curves. We can identify at least 4 factors that explain these differences. To reproduce the theoretical MTFs at the system output, it was necessary to take into account the Transfer Function of the pixels of our detector. However, we do not know precisely their shape, and it was approximated from a simple cardinal sinus with a cut-off frequency at $40 \mathrm{cycle} / \mathrm{mm}$ corresponding to a pixel size of $25 \mu \mathrm{m}$. Secondly, there may be misalignments of the different parts of the kaleidoscope-based system, particularly at the detector level. Indeed, the detector is very close to the mechanics of the rear lens and the amplitude for its alignment is limited. The alignment of the detector is thus delicate, and a defocus or tilt may have been induced. Third, the theoretical MTFs do not consider tolerances. We do not know which deviations from the nominal value have the curvatures or thicknesses of the lenses. Finally, all lenses are in germanium. The refractive index of this material varies greatly with temperature. Fig. 11 shows the same MTF curves for different environmental temperatures. Thus, if the environmental temperatures are different during the alignment and during the measurement, then performances can quickly be degraded. 


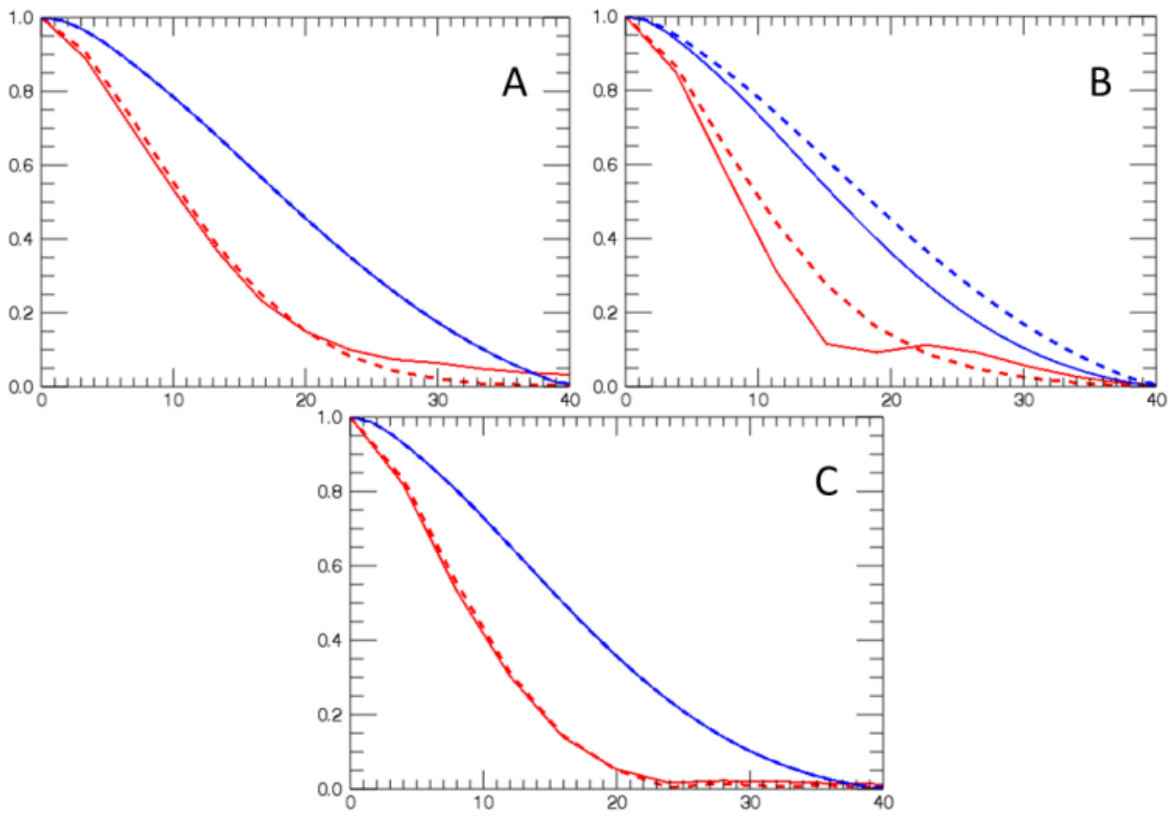

Fig. 10. MTFs for the central field for A) the central subimage, B) a side subimage, C) a diagonal subimage. In blue, the theoretical MTFs, in red the measured MTFs. In full line the tangential MTFs, in dash line the sagittal MTFs...
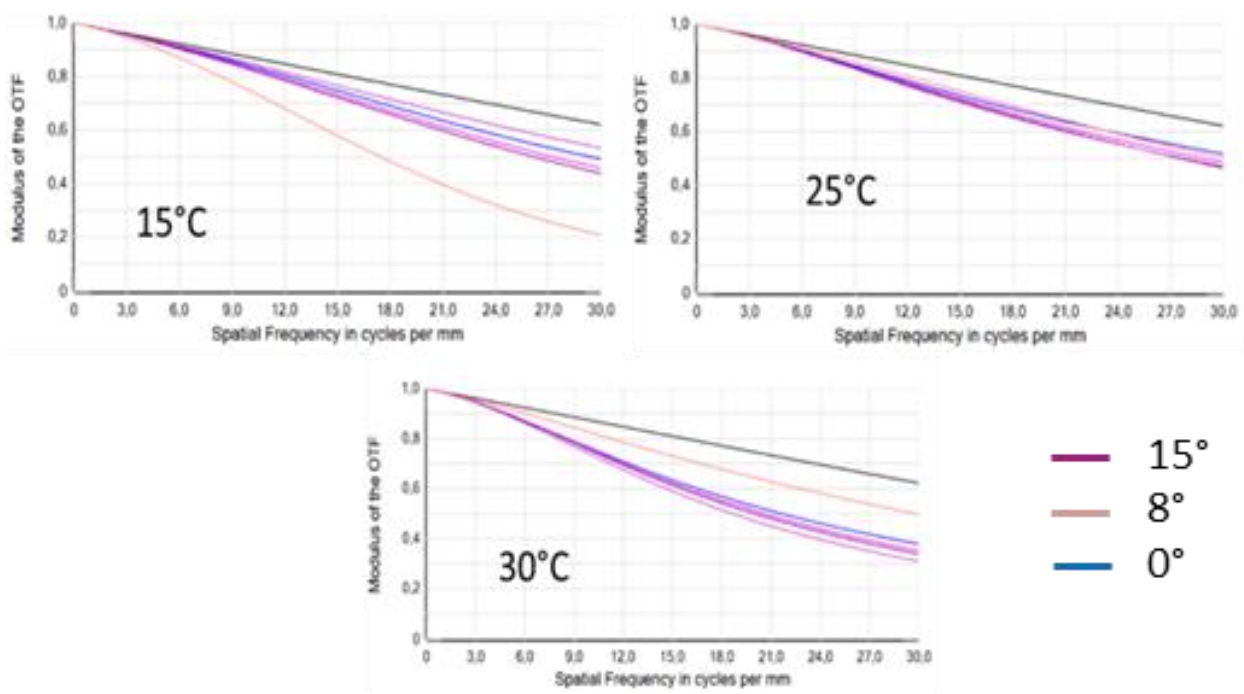

Fig. 11. MTFs of the central subimage for several fields of view and different environmental temperatures.

\section{STUDY OF A TOMBO-BASED SYSTEM WITH A FRONT AFOCAL HAVING THE SAME FEATURES AS THE KALEIDOSCOPE-BASED SYSTEM}

The kaleidoscope-based architecture is obviously less compact than a multi-channel TOMBO architecture with its 110 $\mathrm{mm}$ of length compared to its focal length of $6.5 \mu \mathrm{m}$. In comparison, a two-stage Petzval design for a TOMBO-based system would have a thickness around $10 \mathrm{~mm}$. However, the kaleidoscope architecture provides additional advantages over a TOMBO-based system:

- First, a kaleidoscope architecture makes it possible to exceed the limits of equation 1 by decoupling the field of view and the aperture.

- In addition, it has some flexibilities to modify the field of view by only changing the front lens.

- Finally, there is no risk of crosstalk because the entrance of the kaleidoscope acts as a field stop. 
Therefore, due to these three advantages, it is more appropriate to compare the kaleidoscope architecture with a TOMBO architecture preceded by an afocal used to adapt the field of view of the TOMBO-based system, when comparing the length of both designs. This afocal must have an intermediate image plane to be able to insert a field stop.

An example of a TOMBO-based system with an afocal is shown in Fig. 12. In this example, the afocal transforms a horizontal $30^{\circ}$ field of view into a horizontal $52^{\circ}$ field of view which is in adequacy with a TOMBO-based system opened at F/1 as seen in section 2. Moreover, obtaining square subimages for such a large horizontal field of view is not possible with this architecture. We can see that the TOMBO architecture immediately loses its major advantage of compactness if we want to meet the three advantages previously mentioned. Indeed, it has now a thickness of $55 \mathrm{~mm}$ instead of $10 \mathrm{~mm}$. Moreover, this camera uses 7 lenses by subimages which is fairly the same number of lenses as for a kaleidoscope-based system.

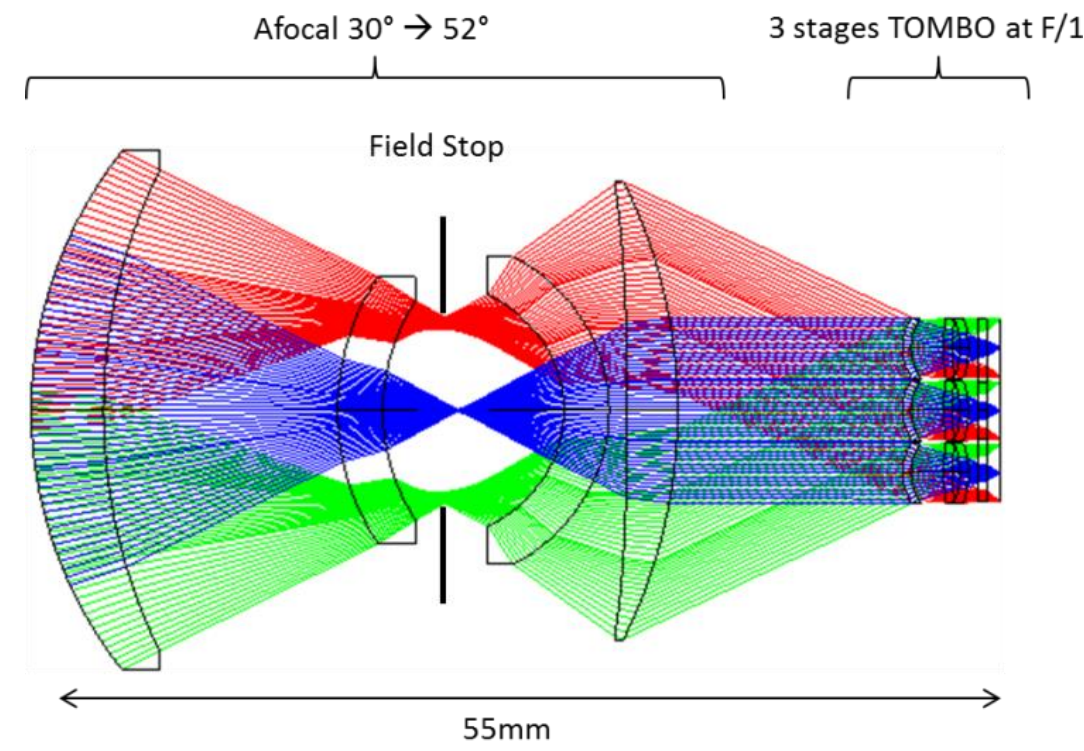

Fig. 12. Zemax design of a TOMBO coupled with an afocal.

\section{STUDY OF THE PARALLAX OF THE KALEIDOSCOPE-BASED SYSTEM AND THE TOMBO-BASED SYSTEM WITH A FRONT AFOCAL}

In this section, we compare the parallax of the kaleidoscope-based system and of the TOMBO-based system with a front afocal. In Fig. 13, we can see a chief ray plot through the kaleidoscope camera and the same thing for the TOMBO camera coupled with an afocal in Fig. 14. We notice in these figures that the parallax is almost identical and is equal to around $12 \mathrm{~mm}$ for the side images. 


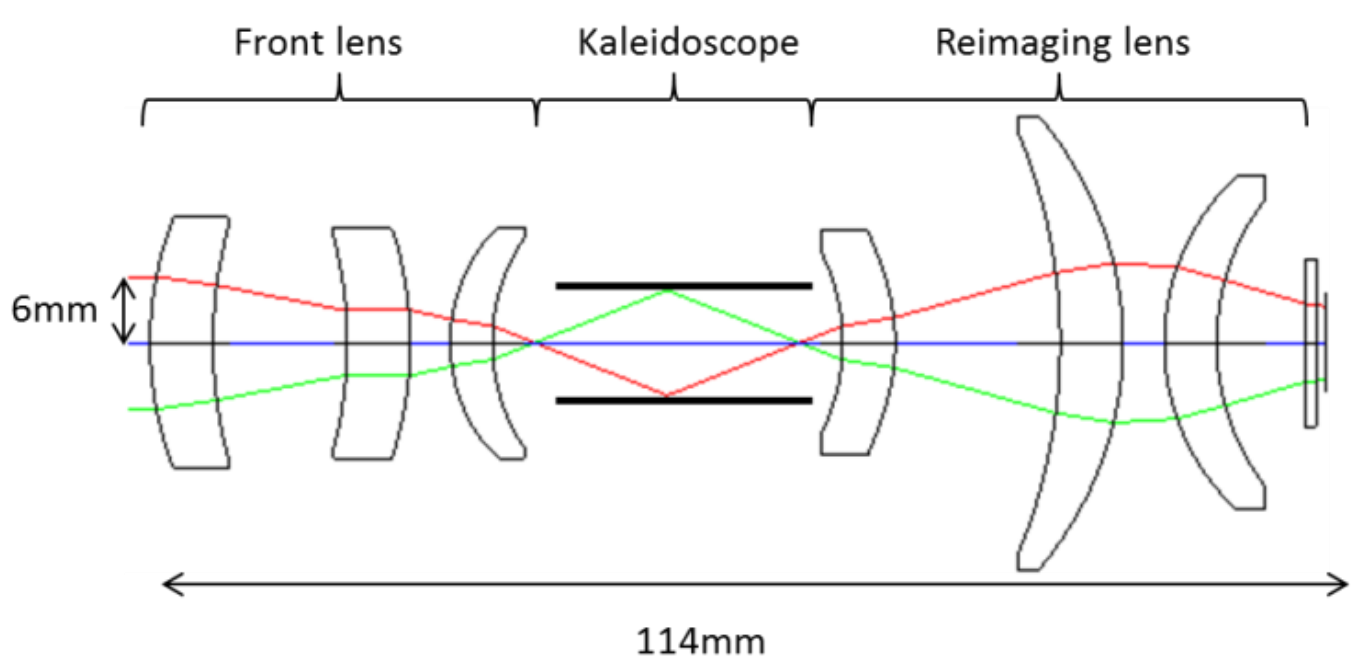

Fig. 13. Chief ray plot through the kaleidoscope camera for the central subimage and two side subimages.

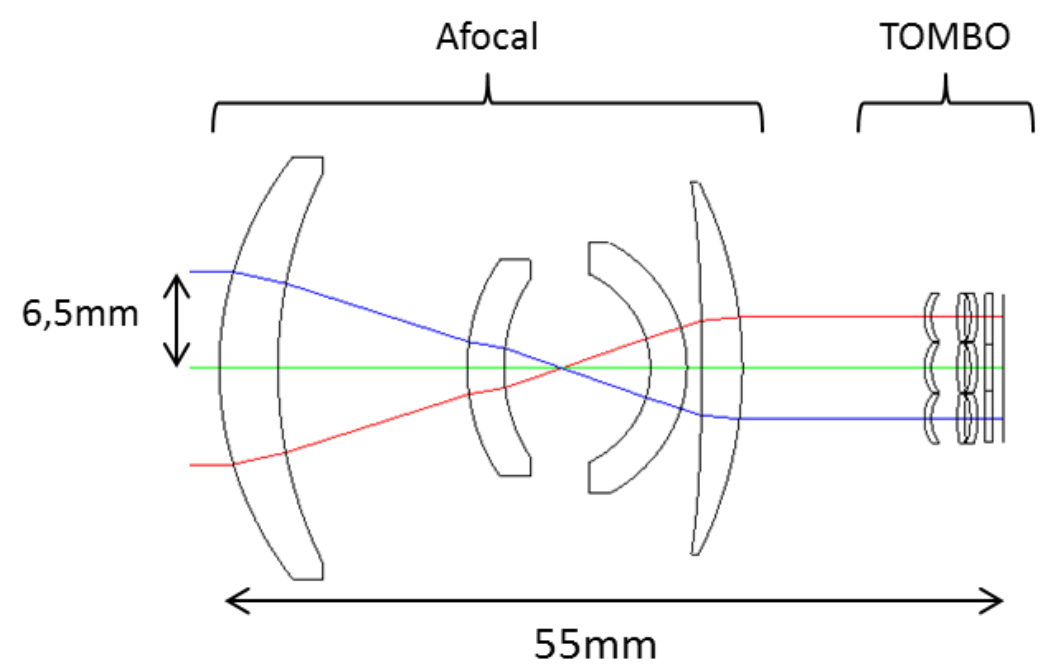

Fig. 14. Chief ray plot through the TOMBO camera coupled with an afocal for the central subimage and two side subimages.

\section{CONCLUSION}

In this paper, we demonstrated an adaptation of a kaleidoscope-based system for an application in the LWIR and compatible with an uncooled infrared detector. A realization of a demonstrator and its characterization were presented. This multi-view system can answer the high numerical aperture challenge required by microbolometers while having a great flexibility with the field of view and while limiting the unused pixels between the subimages. This system only uses conventional components, thus enabling rapid and cost-effective manufacturing and assembling. It also has the advantage of being modular and the field of view can be adapted by only changing the front lens. We thus drew three advantages of the kaleidoscope-based system over a classical TOMBO-based system: FNumber/FOV uncoupling, FOV flexibility and crosstalk free between the subimages thanks to the presence of a field stop. To meet these three advantages, a TOMBO-based system has to add an afocal in front of the TOMBO module and therefore, this system lost its advantage of compactness compared to a kaleidoscope-based system. Moreover, highly aspherical lenslet arrays are unconventional 
components that can be difficult to manufacture and align, and despite this complexity, the resulting subimages cannot be square for high fields of view.

\section{REFERENCES}

[1] Nathan A. Hagen, Michael W. Kudenov, "Review of snapshot spectral imaging technologies," Opt. Eng. 52(9) 090901 (23 September 2013).

[2] I. S. Bowen, "The image slicer, a device for reducing loss of light at slit of stellar spectrograph," Astrophys. J. 88(2),113-124 (1938).

[3] G. Courtès, "Méthodes d'observation et étude de l'hydrogène interstellaire en émission," Annales d'Astrophysique 23(2), 115-244 (1960).

[4] N. S. Kapany, "Fiber optics," in Concepts of Classical Optics, J. Strong, Ed., pp. 553-579, Dover, Mineola, NY (2004).

[5] J. Tanida, T. Kumagai, K. Yamada, S. Miyatake, K. Ishida, T. Morimoto, N. Kondou, D. Miyazaki, and Y. Ichioka, "Thin observation module by bound optics (TOMBO): concept and experimental verification," Appl. Opt. 40, 1806-1813 (2001)).

[6] A. Portnoy, N. Pitsianis, X. Sun, D. Brady, R. Gibbons, A. Silver, R. Te Kolste, C. Chen, T. Dillon, and D. Prather, "Design and characterization of thin multiple aperture infrared cameras," Appl. Opt. 48, 2115-2126 (2009)

[7] R. Horstmeyer, G. Euliss, R. Athale, and M. Levoy, "Flexible multimodal camera using a light field architecture," in Proceedings of the IEEE International Conference on Computational Photography (IEEE, 2009).

[8] K. Cossu, G. Druart, E. Belhaire, F. Champagnat, T. Lépine, "Design, implementation and performance analysis of cooled infrared cameras with single FPA depth estimation capabilities," Proc. of SPIE Vol. 9948 99480B-1

[9] A. Manakov, J. F. Restrepo, O. Klehm, R. Hegedus, E. Eisemann, H. Seidel, and I. Ihrke, “A reconfigurable camera add-on for high dynamic range, multispectral, polarization, and light-field imaging," ACM Trans. Graph. 32, 47:1-47:14 (2013).

[10] S. Pacheco, and R. Liang, "Snapshot, reconfigurable multispectral and multi-polarization telecentric imaging system," Opt. Express 22, 16377-16385 (2014)

[11]F.de la Barrière, G. Druart, N. Guérineau and J. Taboury, " Design strategies to simplify and miniaturize imaging systems," Appl. Opt. 50, 943, (2011)

[12] Guillem Carles, Gonzalo Muyo, Nicholas Bustin, Andrew Wood, and Andrew R. Harvey, "Compact multiaperture imaging with high angular resolution," J. Opt. Soc. Am. A 32, 411-419 (2015)

[13] G. Druart, N. Guérineau, R. Haïdar, E. Lambert, M. Tauvy, S. Thétas, S. Rommeluère, J. Primot and J. Deschamps, " MULTICAM: a miniature cryogenic camera for infrared Detection, " Proc. of SPIE Vol. 6992, 69920G, (2008) 